\title{
Deposition of TiN/CrN hard superlattices by reactive d.c. magnetron sputtering
}

\author{
HARISH C BARSHILIA and K S RAJAM* \\ Surface Engineering Division, National Aerospace Laboratories, Bangalore 560 017, India
}

MS received 26 June 2002; revised 4 December 2002

\begin{abstract}
Multilayer superlattice coatings of $\mathrm{TiN} / \mathrm{CrN}$ were deposited on silicon substrates using a reactive d.c. magnetron sputtering process. Superlattice period, also known as modulation wavelength $(\Lambda)$, was controlled by controlling the dwell time of the substrate underneath $\mathrm{Ti}$ and $\mathrm{Cr}$ targets. $\mathrm{X}$-ray diffraction (XRD), nanoindentation and atomic force microscopy (AFM) were used to characterize the films. The XRD data showed 1st and 2nd order satellite reflections along the principal reflection for films having $132 \AA \geq \Lambda \geq 84 \AA$, thus confirming the formation of superlattice. The multilayer coatings exhibited hardness (H) as high as $3200 \mathrm{~kg} / \mathrm{mm}^{2}$, which is 2 times the rule-of-mixtures value (i.e. $H_{\mathrm{TiN}}=2200 \mathrm{~kg} / \mathrm{mm}^{2}$ and $H_{\mathrm{CrN}}=1000 \mathrm{~kg} / \mathrm{mm}^{2}$ ). Detailed investigations on the effects of various process parameters indicated that hardness of the superlattice coatings was affected not only by modulation wavelength but also by nitrogen partial pressure and ion bombardment during deposition.
\end{abstract}

Keywords. TiN/CrN superlattices; d.c. reactive magnetron sputtering; structural and mechanical properties; nanoindentation; AFM.

\section{Introduction}

In recent years a number of superhard coatings have been developed (Veprek 1999). Nanostructured multilayer coatings of ceramic materials are an emerging class of superhard materials (Yasher and Sproul 1999). In these materials repeating layered structures of two different materials with nanometer-scale dimensions are deposited onto a surface. The nanostructured multilayer coatings are commonly known as superlattices. These materials exhibit exotic properties. The most important feature of superlattice coatings is that the properties can be tailored depending upon the choice, and layer thickness of the constituent materials and deposition parameters. This opens innumerable possibilities to design new materials with desired properties (Sproul 1996). The properties of superlattice coatings greatly affect on the bilayer thickness or modulation wavelength $(\Lambda)$. Enhanced mechanical properties, as compared to single-layered structures, have been reported both for single-crystal superlattices and polycrystalline superlattices. A hardness of $>5000 \mathrm{~kg} / \mathrm{mm}^{2}$ has been reported for single crystalline TiN/NbN and TiN/VN superlattices with a modulation wavelength of $5-8.5 \mathrm{~nm}$, which is much higher than the rule-of-mixtures value (Chu et al 1992).

A variety of mechanisms such as restriction of dislocation motion across the interfaces, strain effects at layer

*Author for correspondence interfaces and differences in shear modulus of superlattice layers have been proposed to explain the observed hardness enhancement in superlattices (Chu and Barnett 1995). TiN/CrN multilayer system is technologically important since it is expected to have higher oxidation resistance apart from the superior mechanical properties. Deposition of multilayer coatings of $\mathrm{TiN} / \mathrm{CrN}$ is a challenging task because the heat of formation of $\mathrm{CrN}$ $(29.8 \mathrm{kcal} / \mathrm{mol})$ is considerably less than that of $\mathrm{TiN}$ $(80.8 \mathrm{kcal} / \mathrm{mol})$. Different partial pressures of nitrogen are, thus, required in the same deposition chamber to deposit stoichiometric $\mathrm{TiN}$ and $\mathrm{CrN}$ coatings. Another biggest problem is that $\mathrm{CrN}$ exists in cubic and hexagonal forms. In general, in order to achieve superlattice structure out of the two materials they should have same crystal structure apart from same lattice parameter and thermal expansion coefficient (Koehler 1970). One needs to address these problems while developing the superlattice coatings of transition metal nitrides. During the deposition of polycrystalline superlattices other issues such as the preferred orientation of the two deposited layers also need to be taken into consideration, which affect the properties of the coatings.

Multilayer coatings are prepared by a variety of physical vapour deposition (PVD) processes, the most common being the reactive sputtering. The properties of the coatings critically depend on the deposition process. With the advancements in the sputtering techniques, researchers use new methods to improve the properties of the multilayer coatings. For example, Yasher et al (1998) 
used an opposed cathode unbalanced magnetron sputtering process to deposit $\mathrm{TiN} / \mathrm{CrN}$ multilayer coatings and demonstrated that coatings exhibited a maximum hardness of $3500 \mathrm{~kg} / \mathrm{mm}^{2}$. The properties of the coatings were greatly dependent on the ion current density. Similarly, Yang et al (2002) used conventional unbalanced magnetron sputtering process and reported that TiN/CrN multilayer coatings exhibited a maximum hardness of $3900 \mathrm{~kg} / \mathrm{mm}^{2}$. The enhanced hardness was attributed to the preferred orientation of the coatings. Likewise, there are several other parameters which affect the properties of the multilayer coatings. It is, therefore, essential to optimize the process conditions for a given deposition technique.

In this article, we describe the growth of TiN/CrN superlattices using reactive d.c. magnetron sputtering process. The superlattice formation of the coatings has been confirmed by X-ray diffraction (XRD) and a nanoindentation technique has been used to measure the mechanical properties. The surface morphology of the coatings has been studied using atomic force microscopy (AFM).

\section{Experimental}

Alternate layers of $\mathrm{TiN}$ and $\mathrm{CrN}$ of varying thicknesses were deposited on silicon substrates using a reactive d.c. magnetron sputtering system. Figure 1 shows the schematic diagram of the sputtering system. The sputtering system consists of ( 3 " diameter) 4 sputtering guns (US Thin Film Products, Inc.). In order to get varying thicknesses of $\mathrm{TiN}$ and $\mathrm{CrN}$ layers, 0.006 meter thick high purity $\mathrm{Ti}(99.95 \%)$ and $\mathrm{Cr}(99.99 \%)$ targets were sputtered for different durations in high purity argon (99.999\%) and nitrogen $(99.999 \%)$ plasma. The power densities were $\sim 5$ and $\sim 2$ watts $/ \mathrm{cm}^{2}$ for $\mathrm{Ti}$ and $\mathrm{Cr}$ targets, respectively. Typically, TiN/CrN multilayers were deposited under a base pressure of $\sim 5.0 \times 10^{-4} \mathrm{~Pa}$ and a total $\mathrm{Ar}+\mathrm{N}_{2}$ gas pressure of $3.0 \times 10^{-1} \mathrm{~Pa}$. The flow

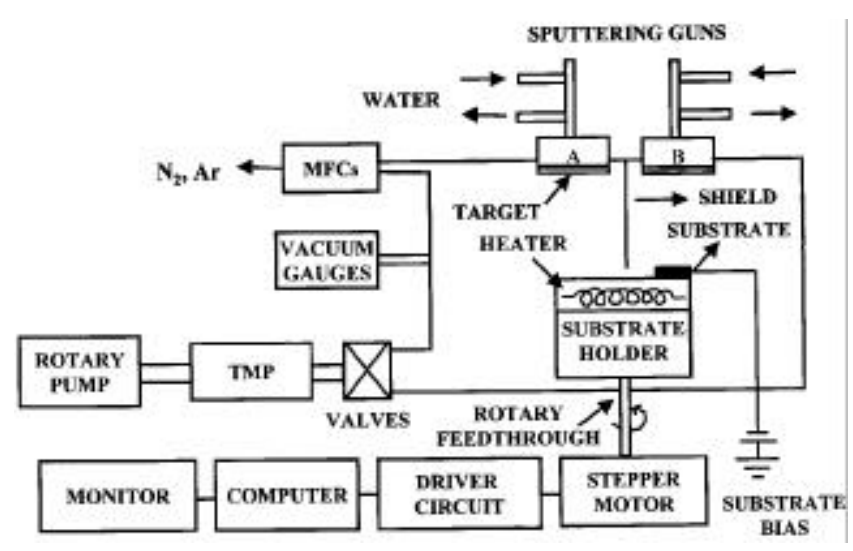

Figure 1. Schematic diagram of the d.c. reactive magnetron sputtering system used to prepare TiN/CrN multilayer coatings. rates of nitrogen and argon were controlled by MKS (model 247) mass flow controllers. Special gas feeding system was designed so as to create the differential partial pressures of the reactive gas near the $\mathrm{Cr}$ and $\mathrm{Ti}$ targets. The substrate to target distance was 0.054 meter. A substrate bias of $-200 \mathrm{~V}$ was applied to improve the mechanical properties of the coatings. This resulted in a current density of 1.1 and $0.3 \mathrm{~mA} / \mathrm{cm}^{2}$, respectively for TiN and CrN sputtered layers. Under these conditions the growth rates were 2 and $6 \AA / s$, respectively for TiN and $\mathrm{CrN}$. Equal number of $\mathrm{TiN}$ and $\mathrm{CrN}$ layers were deposited in all multilayer samples. The total thicknesses of the films were about $1.5 \mu \mathrm{m}$. Samples were deposited at different modulation wavelengths.

In order to deposit multilayer coatings with controlled layer thicknesses and repeatability, a PC based dwell time controller was designed. In this system a stepper motor was connected to the substrate holder through a rotary feedthrough and the stepper motor was controlled by a driver circuit. This allowed controlling the thicknesses of individual layers down to $2 \AA$. Chemically cleaned substrates were heated for out-gassing and then cleaned by plasma bombardment. A $0.5 \mu \mathrm{m}$ thick titanium interlayer was incorporated between the substrate and the film to improve the adhesion of the coating.

The XRD data of the films in glancing-angle geometry were recorded in a Rigaku D/max 2200 powder diffractometer with thin film attachment. The X-ray source was a $\mathrm{CuK}_{\alpha}$ radiation $(\lambda=1.5418 \AA)$. The glancing angle was $1^{\circ}$ for all the measurements. The hardness measurements were performed in a nanoindenter (CSEM Instruments). The applied load was $5 \mathrm{mN}$. Under this load the indentation depth was much less than 1/10th of the coating thickness, thus eliminating the effect of substrate on the hardness measurements. Ten indentations were made on each sample. The hardness was calculated from the loading portion of load vs displacement curves. Surface morphologies of the coatings were investigated by a noncontact probe AFM (surface imaging systems).

\section{Results and discussion}

As mentioned earlier $\mathrm{CrN}$ exists in different phases and more so there is a limited range of nitrogen concentration wherein stoichiometric phases of $\mathrm{TiN}$ and $\mathrm{CrN}$ with $\mathrm{B} 1$ structure exist. So it becomes essential first to optimize the process parameters for the deposition of stoichiometric cubic-TiN and $\mathrm{CrN}$ phases. The deposition conditions were carefully optimized after a series of experiments involving variation of nitrogen partial pressure, target power, operating pressure, substrate bias and current density on the substrate. Detailed discussion on the role of these parameters on the properties of $\mathrm{TiN}$ and $\mathrm{CrN}$ formation will be published later. Typical deposition condi- 
tions for TiN were: sputtering power $=225$ watts, substrate bias $=-200 \mathrm{~V}$, current density on the substrate $=$ $1.1 \mathrm{~mA} / \mathrm{cm}^{2}$, flow rate of nitrogen $=2.0 \mathrm{sccm}$ and operating pressure $=4.0 \times 10^{-1} \mathrm{~Pa}$. Stoichiometric cubic $\mathrm{CrN}$ phase was obtained under the following conditions: sputtering power $=100$ watts, substrate bias $=-200 \mathrm{~V}$, current density on the substrate $=0.3 \mathrm{~mA} / \mathrm{cm}^{2}$, flow rate of nitrogen $=2 \cdot 0 \mathrm{sccm}$ and operating pressure $=4.0 \times 10^{-1} \mathrm{~Pa}$. Under these conditions (111) oriented cubic phases of TiN and $\mathrm{CrN}$ were obtained. The lower panels of figure 2 show the XRD data of $\mathrm{TiN}$ and $\mathrm{CrN}$ coatings. The intensity of (200) orientation for both TiN and CrN was very low. Other reflection planes such as (220) and (311) were completely absent in the diffraction patterns (not plotted in the figure). This shows that under these deposition conditions both $\mathrm{TiN}$ and $\mathrm{CrN}$ coatings were highly oriented along (111) direction.

After optimizing the deposition conditions for TiN and $\mathrm{CrN}$, multilayer samples were prepared at different modulation wavelengths. The top panel of figure 2 shows a typical low-angle XRD pattern of TiN/CrN multilayer coating deposited at a modulation wavelength of $132 \AA$. The individual layer thicknesses were $64 \AA$ each. The structure is composed of one principal reflection, the $d$ value of which lies in between that of $\mathrm{TiN}$ and $\mathrm{CrN}$. The principal reflection is flanked by 1 st and 2 nd order satel-

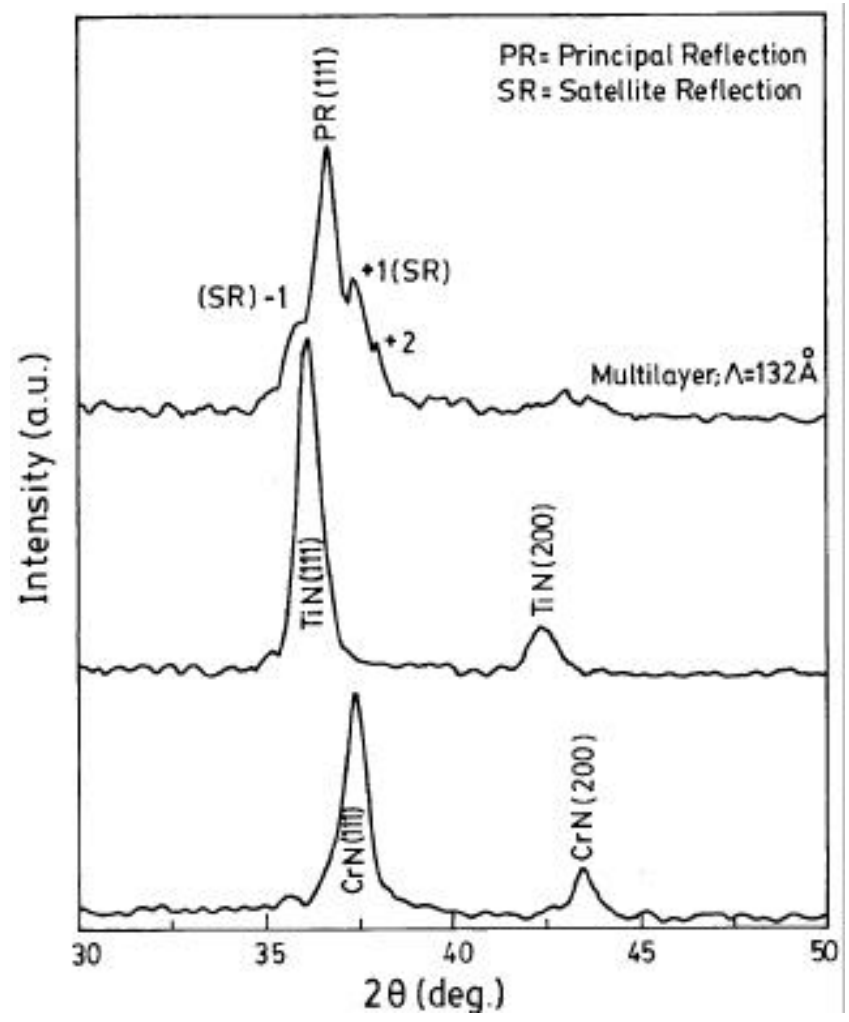

Figure 2. Glancing-angle X-ray diffraction profiles of: (a) single layer $\mathrm{CrN}$, (b) single layer TiN and (c) TiN/CrN multilayer coatings deposited on silicon substrates. lite reflections, which are indicative of superlattice formation under these conditions. The satellite reflections were observed for films having $132 \AA \geq \Lambda \geq 84 \AA$. At very low modulation wavelengths only principal reflection was observed and satellite reflections were absent. This may be due to alloying of the two materials at low $\Lambda$ value (Madan et al 1997). At large modulation wavelengths characteristic reflections of $\mathrm{TiN}$ and $\mathrm{CrN}$ were observed. Because at large $\Lambda$, thicknesses of individual layers are large and the X-rays can identify the component materials.

Load vs displacement curves were measured for single layer TiN and $\mathrm{CrN}$, and $\mathrm{TiN} / \mathrm{CrN}$ multilayer samples. Typical plots obtained for three samples are shown in figure 3. After initial contact of the indenter on surface, the load was increased at a predetermined rate $(10 \mathrm{mN} /$ min) to the desired maximum load $(5 \mathrm{mN})$ and then decreased at the same rate $(10 \mathrm{mN} / \mathrm{min})$ to zero. The unloading curve followed the partial elastic recovery of the sample material. From this plot the hardness was calculated using Oliver and Pharr (1992) method. The area formed by loading and unloading curves, defined as plastic deformation work, $W_{\mathrm{p}}$, can be used to assess the resistance of plastic deformation and the wear resistance of the coatings. The resistance of plastic deformation is inversely proportional to the plastic deformation work. In the present study, TiN/CrN multilayer coating shows the smallest plastic deformation work and the largest resis-

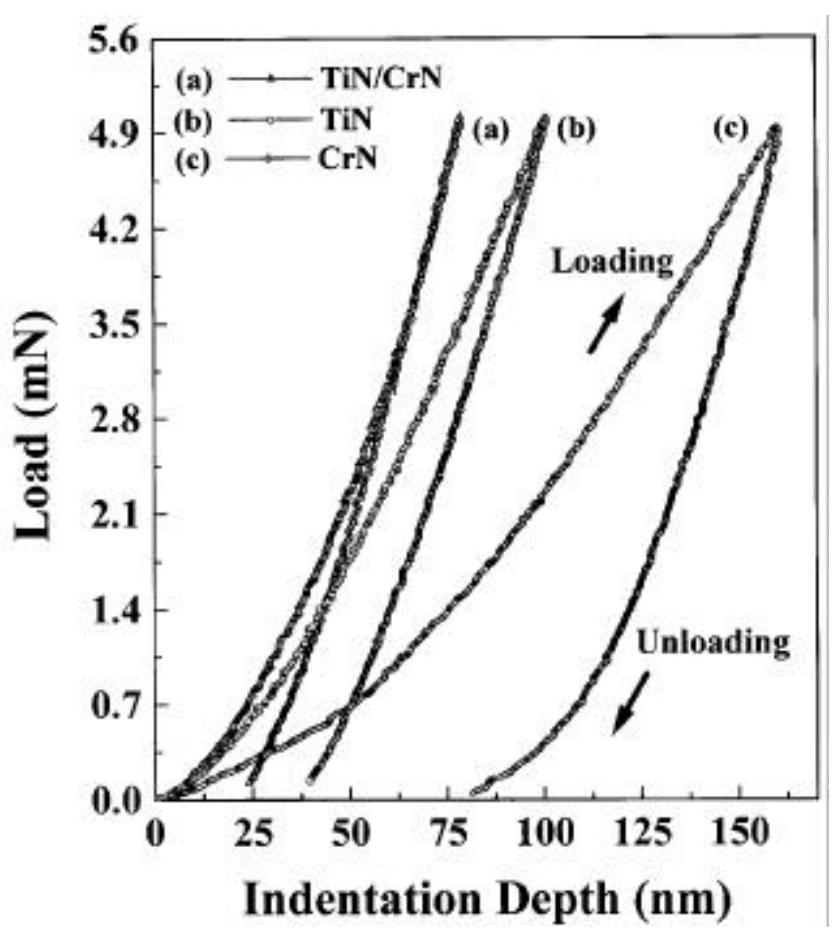

Figure 3. Schematic representations of load vs displacement curves for (a) a typical TiN/CrN multilayer coating, (b) TiN coating and (c) CrN coating. 
tance to plastic deformation as compared to the single layer $\mathrm{TiN}$ and $\mathrm{CrN}$ coatings.

For the softest film (i.e. $\mathrm{CrN}$ ), the maximum indentation depth was $160 \mathrm{~nm}$, which is about 1/10th of the coating thickness. For the hardest film (i.e. TiN/CrN), the maximum indentation depth was only $75 \mathrm{~nm}$. The hardness values obtained from these plots were 1000, 2200 and $3200 \mathrm{~kg} / \mathrm{mm}^{2}$, respectively for $\mathrm{CrN}$, TiN and TiN/ $\mathrm{CrN}$ multilayer coatings. The rule-of-mixtures value for TiN and CrN would be about $1600 \mathrm{~kg} / \mathrm{mm}^{2}$. The observed hardness enhancement in $\mathrm{TiN} / \mathrm{CrN}$ multilayer coatings is, thus, about 2 times the rule-of-mixtures value. Similar observations have been reported in the literature for $\mathrm{TiN} / \mathrm{NbN}$ and $\mathrm{TiN} / \mathrm{VN}$ multilayer coatings (Chu et al 1992). The hardness for multilayer was dependent on modulation wavelength. For example, the observed hardness values were 2800 and $2500 \mathrm{~kg} / \mathrm{mm}^{2}$ at $\Lambda=50$ and $400 \AA$, respectively. At very low and very high $\Lambda$ the hardness got decreased because of the loss of the superlattice structure under these conditions. This is supported by XRD data, which showed the loss of superlattice structure for the films having $64 \AA \geq \Lambda \geq 150 \AA$. For $132 \AA \geq \Lambda \geq 84 \AA$, the hardness was almost constant.

The enhancement in the hardness of TiN/CrN multilayer coatings at low modulation wavelengths (132 $\geq \Lambda \geq 84 \AA$ ) is attributed to the dislocations. As the thicknesses of the individual layers are very small, generation of dislocations (such as Frank Read) cannot take place inside a given layer (Koehler 1972). Even if the dislocations are generated in a layer they cannot propagate along the growth direction because the interfaces act as the barrier for the propagation of dislocations. Furthermore, there is a large difference between the dislocation line energies for $\mathrm{TiN}$ and $\mathrm{CrN}$ because of large difference between the shear moduli of TiN (192 GPa) and $\mathrm{CrN}$ (125 GPa). This prevents the dislocation glide across the interfaces and hence pinning of dislocations at the interfacial sites. These two factors are believed to be responsible for the enhanced mechanical properties of the multilayer coatings.

One of the most important features of the superlattices is the sharp interface. A superlattice having diffused interfaces will exhibit poor mechanical properties, whereas sharp interfaces will minimize the intermixing of the two materials at the interfaces and will exhibit superior properties (Sproul 1996). The nature of interface depends on the interdiffusion of the two materials and roughness of the deposited layers. As the coatings were deposited at very low temperature $\left(<100^{\circ} \mathrm{C}\right)$ interdiffusion between TiN and $\mathrm{CrN}$ can be neglected. Thus roughness of the coatings will determine the nature of the interfaces. The roughness largely depends on the growth rate, and energy and flux of the impinging ions depositing on the substrate. In the present work, these parameters were judiciously controlled to achieve nano-smooth layers. Though the cross-sectional transmission electron micro-

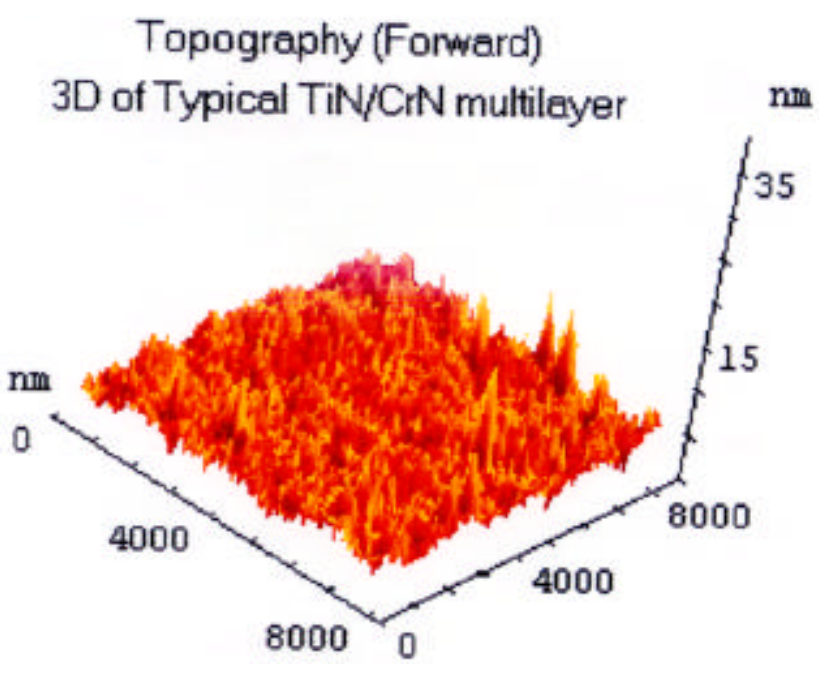

Figure 4. 3-dimensional AFM image of a typical TiN/CrN multilayer coating.

scope image will provide the accurate picture about the interfaces, an indirect method would be the investigation of surfaces by AFM. A three-dimensional AFM image of a typical $1.5 \mu \mathrm{m}$ thick $\mathrm{TiN} / \mathrm{CrN}$ multilayer coating is shown in figure 4 indicating an average roughness of $5 \mathrm{~nm}$.

\section{Conclusions}

TiN/CrN multilayer coatings were deposited on silicon substrates using d.c. magnetron sputtering process. At a nitrogen flow rate of $2 \mathrm{sccm}$ and a substrate bias of $-200 \mathrm{~V}$, both TiN and CrN coatings exhibited cubic B1 structure. The XRD data of the multilayer films deposited at $132 \AA \geq \Lambda \geq 84 \AA$ showed a prominent principal reflection along (111) plane, which was flanked by 1 st and 2nd order satellite reflections, thus confirming the formation of superlattice structure. Nanoindentation data showed that for the TiN/CrN multilayer film prepared at $\Lambda=132 \AA$ the hardness was as high as $3200 \mathrm{~kg} / \mathrm{mm}^{2}$, which was $\sim 2$ times the rule-of-mixtures value. The loading and unloading curves of nanoindentation data indicated that $\mathrm{TiN} / \mathrm{CrN}$ exhibited largest resistance to plastic deformation as compared to the single layer TiN and $\mathrm{CrN}$ coatings. The AFM images of $1.5 \mu \mathrm{m}$ thick $\mathrm{TiN} / \mathrm{CrN}$ multilayer exhibited surface roughness on nanometer scale.

\section{Acknowledgements}

The authors thank Dr Anjana Jain for recording the XRD patterns. This research was supported by the Department of Science and Technology and the Council of Scientific and Industrial Research, New Delhi. 


\section{References}

Chu X and Barnett S 1995 J. Appl. Phys. 774403

Chu X, Wong M S, Sproul W D, Rohde S L and Barnett S A 1992 J. Vac. Sci. Technol. A10 1604

Koehler J S 1970 Phys. Rev. B2 547

Madan A, Yashar P, Shinn M and Barnett S A 1997 Thin Solid Films 302147
Oliver W C and Pharr G M 1992 J. Mater. Res. 71564

Sproul D S 1996 Science 273889

Veprek S 1999 J. Vac. Sci. Technol. A17 2401

Yang Q, He C, Zhao L R and Immarigeon J P 2002 Scr. Mater. 46293

Yashar P C and Sproul W D 1999 Vacuum 55179

Yashar P, Barnett S A, Rechner J and Sproul W D 1998 J. Vac. Sci. Technol. A16 2913 